\title{
Suppliment of
}

\section{The development and evaluation of a hydrological seasonal forecast}

system prototype for predicting spring flood volumes in Swedish rivers

$5 \quad$ K. Foster ${ }^{1,2}$, C.B. Uvo ${ }^{2}$, J. Olsson ${ }^{1}$

${ }^{1}$ Research \& Development (hydrology), Swedish Meteorological and Hydrological Institute, 60176 Norrköping, Sweden

${ }^{2}$ Department of Water Resources Engineering, Lund University, Box 118, 22100 Lund, Sweden

Correspondence to: Kean Foster (kean.foster@smhi.se) 


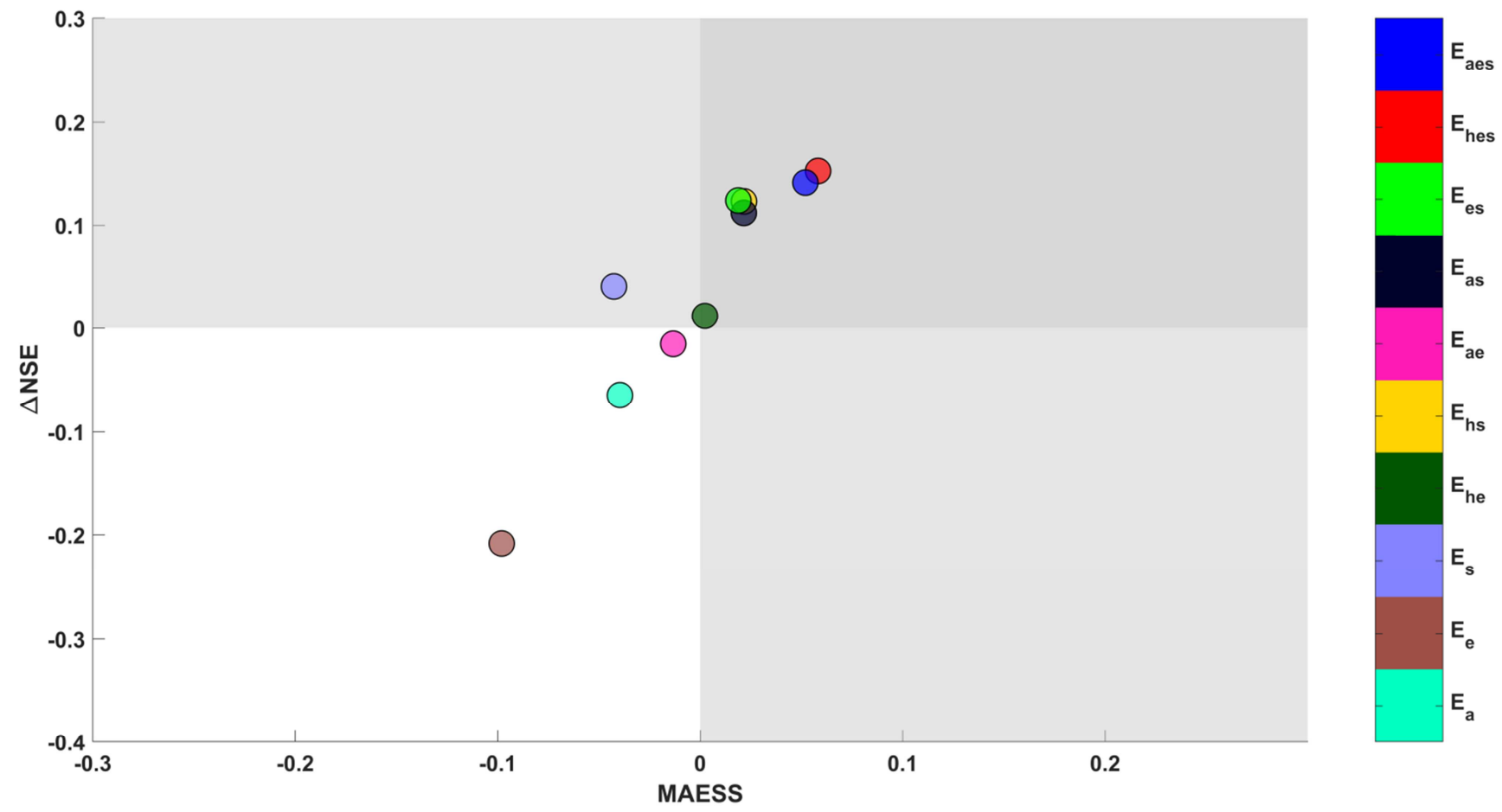

Figure 1. A scatterplot of the MAESS vs $\triangle$ NSE scores for the different combinations of the different individual modelling chains. The shaded quadrants denote the respective areas of skill for the different scores. 

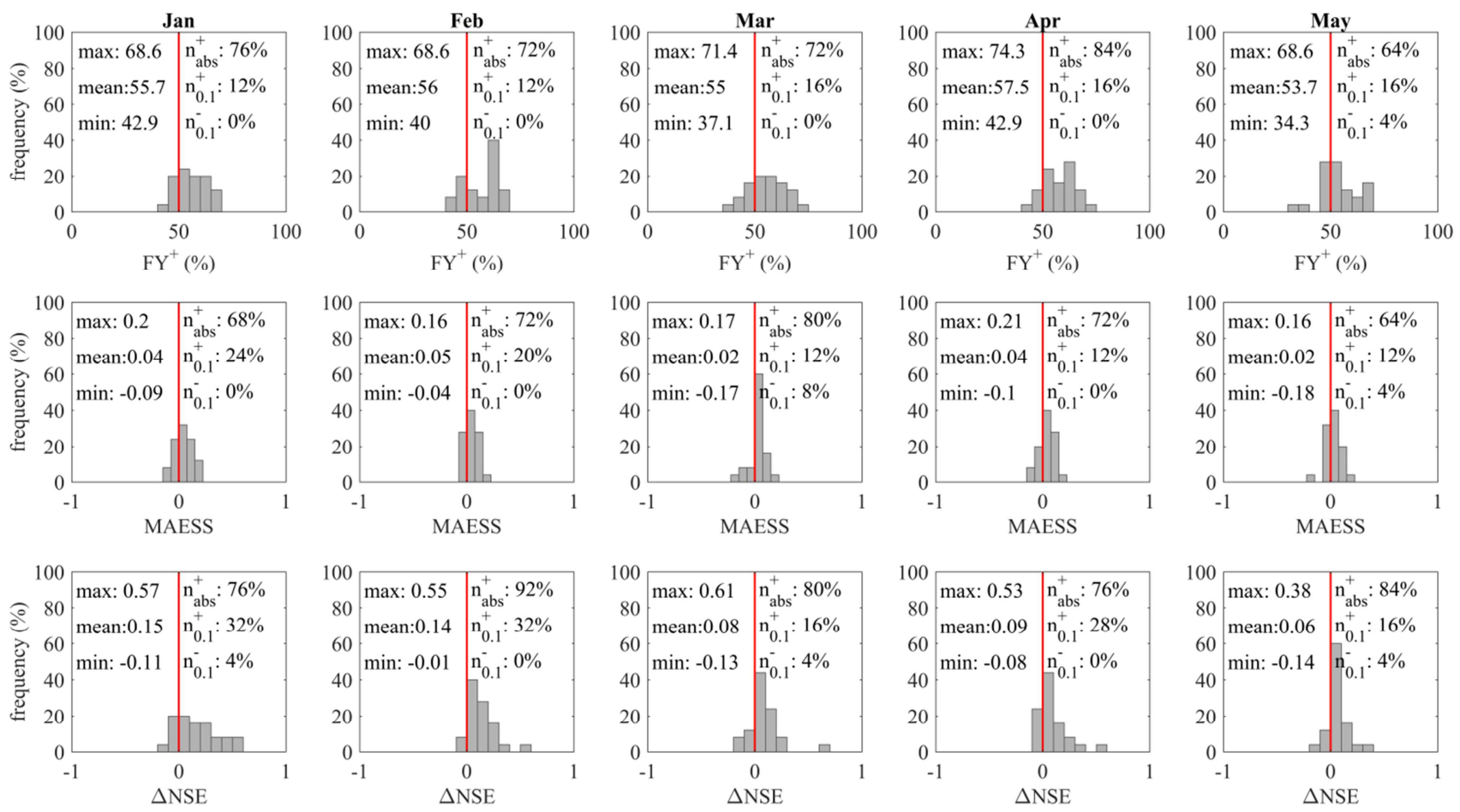

Figure 2. Bootstrapped $(\mathrm{N}=10000) \mathrm{FY}+$, MAESS, and $\triangle \mathrm{NSE}$ scores for $\mathrm{ME}_{\mathrm{hds}}$ with respect to $\mathrm{HE}$ for all subbasins in the cluster $S^{1}$. Each subplot is a histogram of the medians of the bootstrapped validations scores for each initialisation month. Above the histograms are six related statistics: (left of the red line) the maximum, mean, and minimum of the validation scores shown in the

5 histograms; (right of the red line) percentages of the subbasins where $\mathrm{ME}_{\text {hds }}$ performed better than $\mathrm{HE}\left(\mathrm{n}_{\mathrm{abs}}^{+}\right)$, the percentage of subbasins where $M E_{h d s}$ performed better than $\mathrm{HE}\left(\mathrm{n}_{0.1}^{+}\right)$at the significance level 0.1 , and lastly the percentage of subbasins where $\mathrm{ME}_{\mathrm{hds}}$ performed worse than $\mathrm{HE}\left(\mathrm{n}_{\mathbf{0 . 1}}^{-}\right)$at the 0.1 level. 

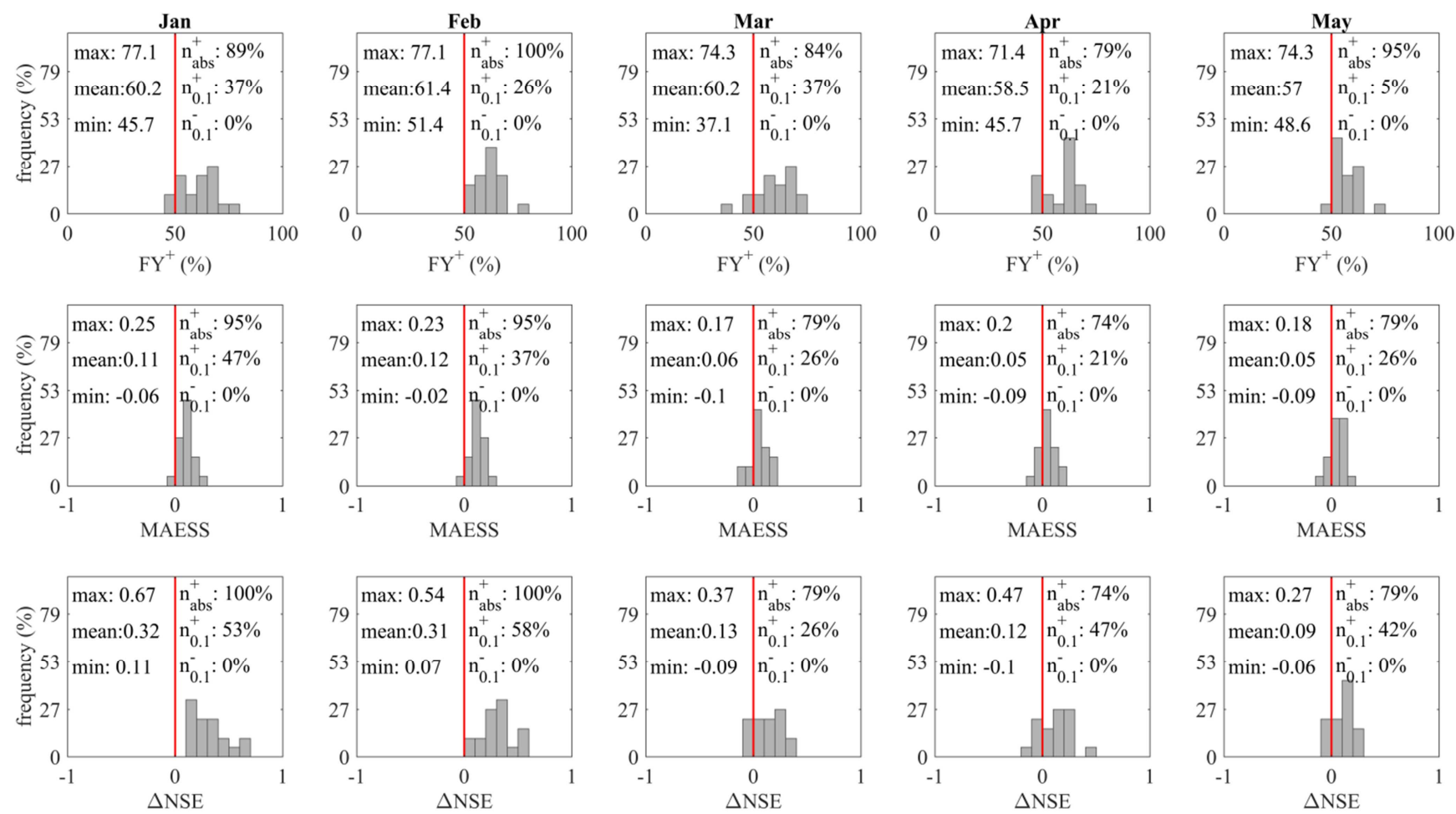

Figure 3. Bootstrapped $(\mathrm{N}=10000) \mathrm{FY}+$, MAESS, and $\triangle \mathrm{NSE}$ scores for $\mathrm{ME}_{\mathrm{hds}}$ with respect to $\mathrm{HE}$ for all subbasins in the cluster $S^{2}$. Each subplot is a histogram of the medians of the bootstrapped validations scores for each initialisation month. Above the histograms are six related statistics: (left of the red line) the maximum, mean, and minimum of the validation scores shown in the

5 histograms; (right of the red line) percentages of the subbasins where $\mathrm{ME}_{\mathrm{hds}}$ performed better than $\mathrm{HE}\left(\mathrm{n}_{\mathrm{abs}}^{+}\right)$, the percentage of subbasins where $M E_{h d s}$ performed better than $\mathrm{HE}\left(\mathrm{n}_{0.1}^{+}\right)$at the significance level 0.1 , and lastly the percentage of subbasins where $\mathrm{ME}_{\mathrm{hds}}$ performed worse than $\mathrm{HE}\left(\mathrm{n}_{0.1}^{-}\right)$at the 0.1 level. 

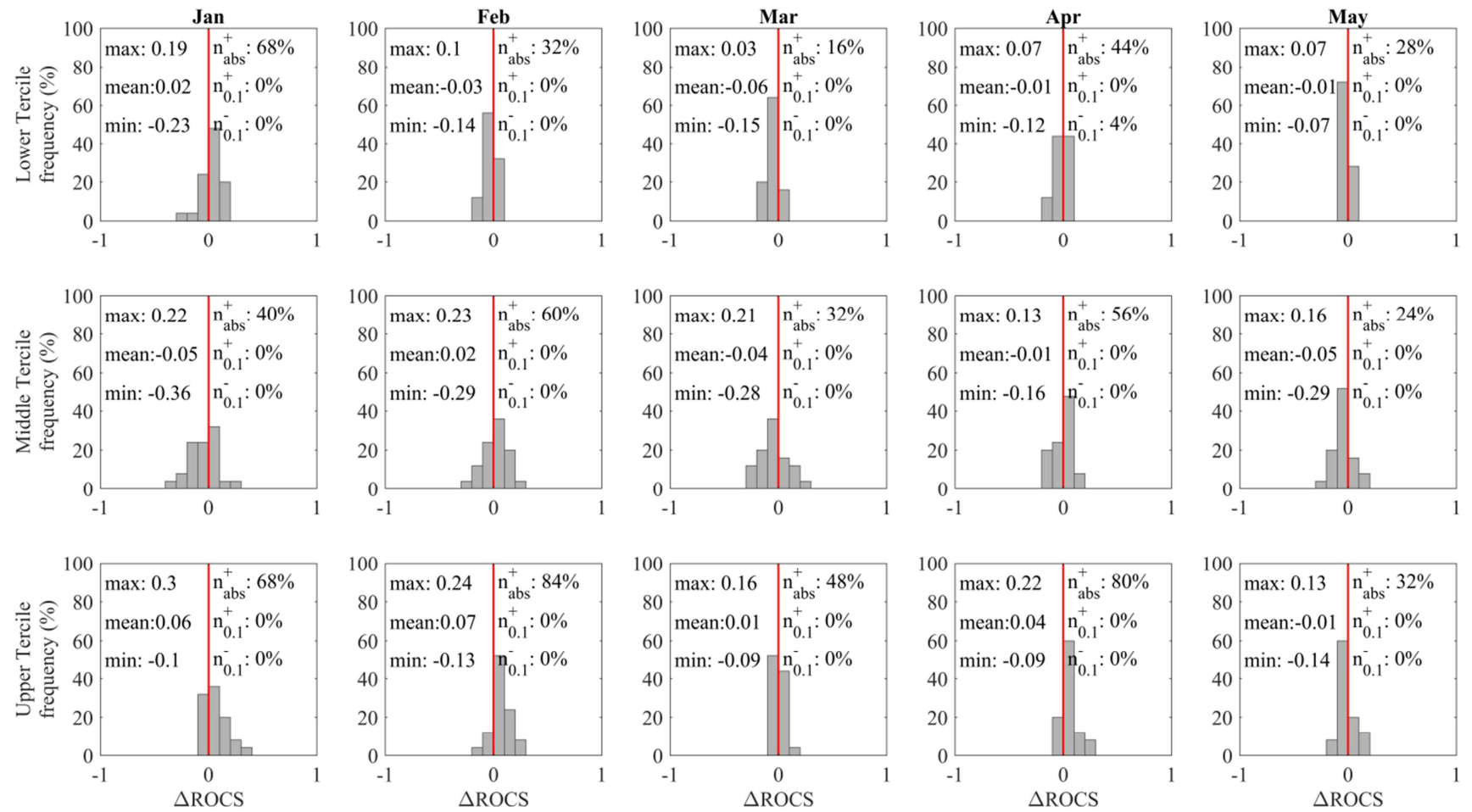

Figure 4. Bootstrapped $(\mathrm{N}=10000) \triangle \mathrm{ROCSS}$ for the lower, middle, and upper terciles between the $\mathrm{ME}_{\mathrm{hds}}$ and $\mathrm{HE}$ for subbasins in the cluster $\mathbf{S}^{\mathbf{1}}$. Each subplot is a histogram of the medians of the bootstrapped validation score's ensembles for each initialisation month. Above the histograms are six related statistics: (left of the red line) the maximum, mean, and minimum of the validation 5 scores shown in the histograms; (right of the red line) percentages of the subbasins where $\mathrm{ME}_{\text {hds }}$ performed better than HE $\left(\mathrm{n}_{\text {abs }}^{+}\right)$, the percentage of subbasins where $\mathrm{ME}_{\mathrm{hds}}$ performed better than $\mathrm{HE}\left(\mathrm{n}_{0.1}^{+}\right)$at the significance level 0.1 , and lastly the percentage of subbasins where $\mathrm{ME}_{\mathrm{hds}}$ performed worse than $\mathrm{HE}\left(\mathrm{n}_{\mathbf{0 . 1}}^{-}\right)$at the 0.1 level. 

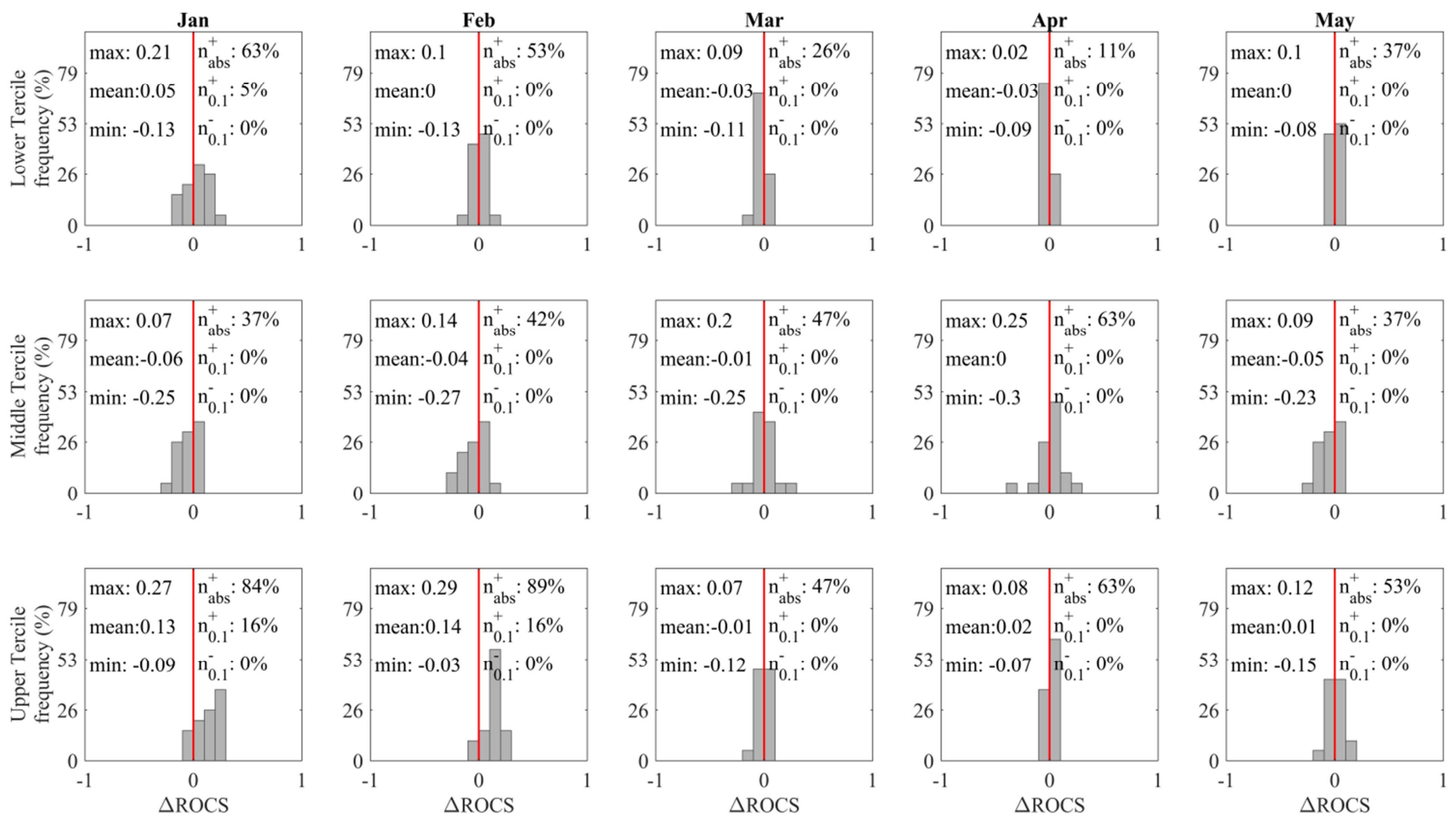

Figure 5. Bootstrapped $(N=10000) \triangle R O C S S$ for the lower, middle, and upper terciles between the $M_{\mathrm{hds}}$ and $\mathrm{HE}$ for subbasins in the cluster $S^{2}$. Each subplot is a histogram of the medians of the bootstrapped validation score's ensembles for each initialisation month. Above the histograms are six related statistics: (left of the red line) the maximum, mean, and minimum of the validation 5 scores shown in the histograms; (right of the red line) percentages of the subbasins where $\mathrm{ME}_{\mathrm{hds}}$ performed better than $\mathrm{HE}\left(\mathrm{n}_{\mathrm{abs}}^{+}\right.$), the percentage of subbasins where $\mathrm{ME}_{\mathrm{hds}}$ performed better than $\mathrm{HE}\left(\mathrm{n}_{0.1}^{+}\right)$at the significance level 0.1 , and lastly the percentage of subbasins where $\mathrm{ME}_{\mathrm{hds}}$ performed worse than $\mathrm{HE}\left(\mathrm{n}_{0.1}^{-}\right)$at the 0.1 level. 